\title{
Exploration of Hygromycin B Biosynthesis Utilizing CRISPR- Cas9-Associated Base Editing
}

Sicong Li, ${ }^{\dagger}$ Qian Liu, ${ }^{\dagger}$ Zhiyu Zhong, Zixin Deng and Yuhui Sun*

Key Laboratory of Combinatorial Biosynthesis and Drug Discovery (Wuhan University), Ministry of Education, and Wuhan University School of Pharmaceutical Sciences, Wuhan 430071, People's Republic of China

†S.L. and Q.L. contributed equally to this work.

* To whom correspondence should be addressed. E mail: yhsun@whu.edu.cn 


\section{TABLE OF CONTENTS}

\section{TABLES}

Table S1. Strains Used in This Study

Table S2. Plasmids Used in This Study

Table S3. Oligonucleotide Primers Used in This Study

\section{FIGURES}

Figure S1. LC-ESI-HRMS and MS/MS Analysis of Hygromycin B and Related Compounds

a. LC-ESI-HRMS and MS/MS Analysis of Hygromycin B (1)

b. LC-ESI-HRMS and MS/MS Analysis of 3-N-methyl-talamine (3)

c. LC-ESI-HRMS and MS/MS Analysis of 3-N-methyl-galacamine (7)

d. LC-ESI-HRMS and MS/MS Analysis of Talamine (4)

e. LC-ESI-HRMS and MS/MS Analysis of 3-N-demethyl-hygromycin B (2)

f. LC-ESI-HRMS and MS/MS Analysis of Hygromycin C (9)

g. LC-ESI-HRMS and MS/MS Analysis of 3- $N$-demethyl-hygromycin C (10)

h. LC-ESI-HRMS and MS/MS Analysis of C-6"-deaminated-C-6"-hydroxylhygromycin $\mathrm{C}(\mathbf{5})$

i. LC-ESI-HRMS and MS/MS Analysis of C-6"-deaminated-C-6"-hydroxylhygromycin B (6)

j. LC-ESI-HRMS and MS/MS Analysis of Galacamine (8)

k. LC-ESI-HRMS and MS/MS Analysis of 2'-epi-hygromycin C (11)

Figure S2. SDS-PAGE Analysis of Purified Recombinant Proteins Used in This Study 
Table S1. Strains Used in This Study

\begin{tabular}{llc}
\hline \multicolumn{1}{c}{ Strain } & \multicolumn{1}{c}{ Characteristic } & Reference \\
\hline Escherichia coli & Host for general DNA manipulation & Invitrogen \\
DH10B & Donor strain for conjugation between E. coli & {$[4]$} \\
ET12567/pUZ8002 & and Streptomyces & \\
BL21(DE3) & Host for recombinant protein expression & Invitrogen \\
Streptomyces hygroscopicus subsp. hygroscopicus & \\
DSM 40578 & Hygromycin B producing wild-type strain & CGMCC \\
$\Delta$ hygD & Mutant with hygD inactivated by base editing & This study \\
$\Delta$ hygJ & Mutant with hygJ inactivated by base editing & This study \\
$\Delta$ hygL & Mutant with hygL inactivated by base editing & This study \\
$\Delta$ hygY & Mutant with hygY inactivated by base editing & This study \\
$\Delta$ hygM & Mutant with hygM inactivated by base editing & This study \\
\hline
\end{tabular}


Table S2. Plasmids Used in This Study

\begin{tabular}{|c|c|c|}
\hline Plasmid & Description & Reference \\
\hline pWHU77 & plB139 derivative with $\mathrm{Tsr}^{\mathrm{R}}$ and $\mathrm{Amp}^{\mathrm{R} *}$ & [1] \\
\hline pSCBE3 & $\begin{array}{l}\text { Source of base editing elements and } \\
\text { sgRNA cassette }\end{array}$ & [2] \\
\hline pWHU77-BE & Vector without sgRNA expression cassette & This study \\
\hline pWHU77-BE-hygD & $\begin{array}{l}\text { Plasmid used for hygD inactivation by base } \\
\text { editing }\end{array}$ & This study \\
\hline pWHU77-BE-hygJ & $\begin{array}{l}\text { Plasmid used for hygJ inactivation by base } \\
\text { editing }\end{array}$ & This study \\
\hline pWHU77-BE-hygL & $\begin{array}{l}\text { Plasmid used for hygL inactivation by base } \\
\text { editing }\end{array}$ & This study \\
\hline pWHU77-BE-hygY & $\begin{array}{l}\text { Plasmid used for hyg } Y \text { inactivation by base } \\
\text { editing }\end{array}$ & This study \\
\hline pWHU77-BE-hygM & $\begin{array}{l}\text { Plasmid used for hygM inactivation by base } \\
\text { editing }\end{array}$ & This study \\
\hline pET28a $(+)$ & Vector for recombinant protein expression & Invitrogen \\
\hline pWHU2857 & Plasmid used for HygM protein expression & [3] \\
\hline pWHU2861 & Plasmid used for HygF protein expression & [3] \\
\hline pET28a(+)-hygY & Plasmid used for HygY protein expression & This study \\
\hline
\end{tabular}


Table S3. Oligonucleotide Primers Used in This Study

\begin{tabular}{|c|c|}
\hline Primer & Oligonucleotide sequences ( $5^{\prime}$ to $3^{\prime}$ ) \\
\hline 77-BE-1 & GGCTCCTTTTGGAGCCTTTTTTTAGCCTGGGGTGCCTAATGAGTG \\
\hline 77-BE-2 & GACTTCCGCCTGCAGGGCCAGGCACTGGCCGTCGTTTTAC \\
\hline sg-cassette-1 & TGCAGGTCGACTCTAGCTAGTGTTCACATTCGAACGGTCTCT \\
\hline sg-cassette-2 & ATCGGGCCCTGGCCAGCTAGGCTACAACTCCTGAGGCTACA \\
\hline BE-hygD-1 & CCTCGGAGTACGAGTGGTGCGGCCACGACTTTACAACACC \\
\hline BE-hygD-2 & GCACCACTCGTACTCCGAGGGTTTTAGAGCTAGAAATAGCAAG \\
\hline BE-hygJ-1 & CCAGGTTGTACACCTGGCCGGGCCACGACTTTACAACACC \\
\hline BE-hygJ-2 & CGGCCAGGTGTACAACCTGGGTTTTAGAGCTAGAAATAGCAAG \\
\hline BE-hygL-1 & TGGTGGCGAAGTACGAGAAGGGCCACGACTTTACAACACC \\
\hline BE-hygL-2 & CTTCTCGTACTTCGCCACCAGTTTTAGAGCTAGAAATAGCAAG \\
\hline BE-hygY-1 & CGGCTGCGCGTCCTGCAGTCGGCCACGACTTTACAACACC \\
\hline BE-hygY-2 & GACTGCAGGACGCGCAGCCGGTTTTAGAGCTAGAAATAGCAAG \\
\hline BE-hygM-1 & TGGAAGAGCGTCCCCTGAGCGGCCACGACTTTACAACACC \\
\hline BE-hygM-2 & GCTCAGGGGACGCTCTTCCAGTTTTAGAGCTAGAAATAGCAAG \\
\hline CK-hygD-1 & GAGGTGGTCACGCACATGAGCG \\
\hline CK-hygD-2 & AGGGCGCGGAGCACCTGCTCGTAC \\
\hline CK-hygJ-1 & TGGGTCTACAACGCCATGCCGGT \\
\hline CK-hygJ-2 & TCGCGGCCAGTTGGGTGCGCAGT \\
\hline CK-hygL-1 & CGGCTGGTGGACGTGGAGCCGG \\
\hline CK-hygL-2 & CAGCACGGTGTGATGGCTGCG \\
\hline CK-hygY-1 & GCTGCCGTTCCGGGACGACTTCG \\
\hline CK-hygY-2 & CCAGAAGTCGTCCCGCATCCTCG \\
\hline CK-hygM-1 & GAGCCCGAGCGGATGGGCGAGATCG \\
\hline CK-hygM-2 & CTTCCCAAAGGTGTCGATGCGCT \\
\hline PR-hygY-1 & GCCGCGCGGCAGCCATATGAGTTATGGCCTCGTCGACGGG \\
\hline PR-hygY-2 & CGACGGAGCTCGAATTCAGAACCTCTTTCCCTGGGCGTAG \\
\hline
\end{tabular}


Figure S1. LC-ESI-HRMS and MS/MS Analysis of Hygromycin B and Related Compounds

a. LC-ESI-HRMS and MS/MS Analysis of Hygromycin B (1)

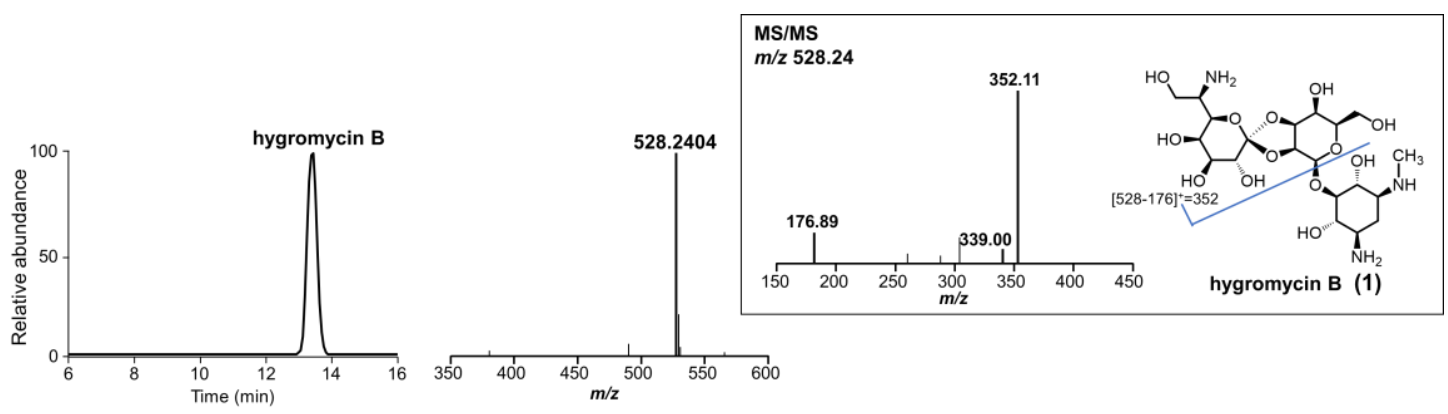

b. LC-ESI-HRMS and MS/MS Analysis of 3- $N$-methyl-talamine (3)
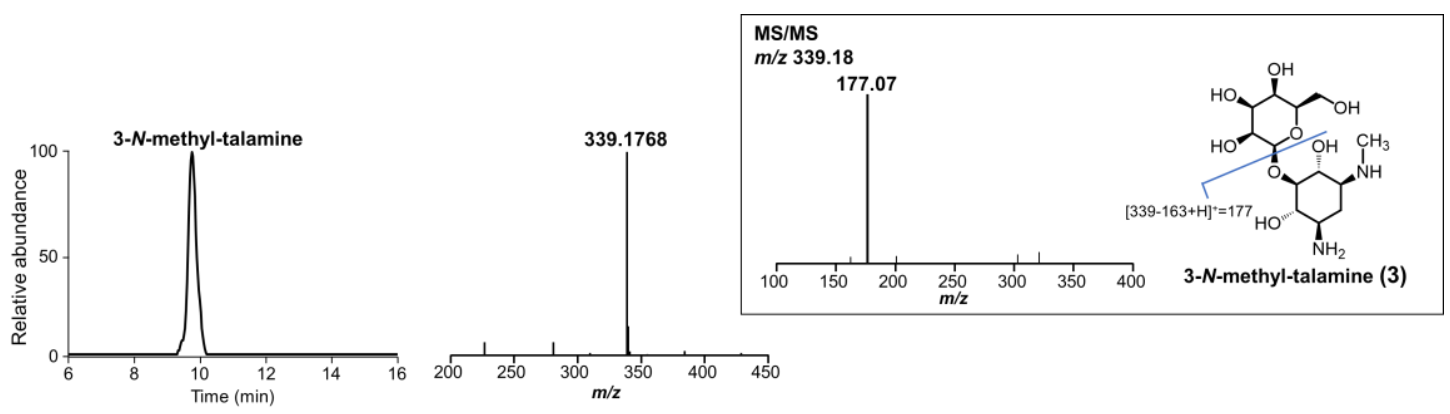

C. LC-ESI-HRMS and MS/MS Analysis of 3-N-methyl-galactamine (7)
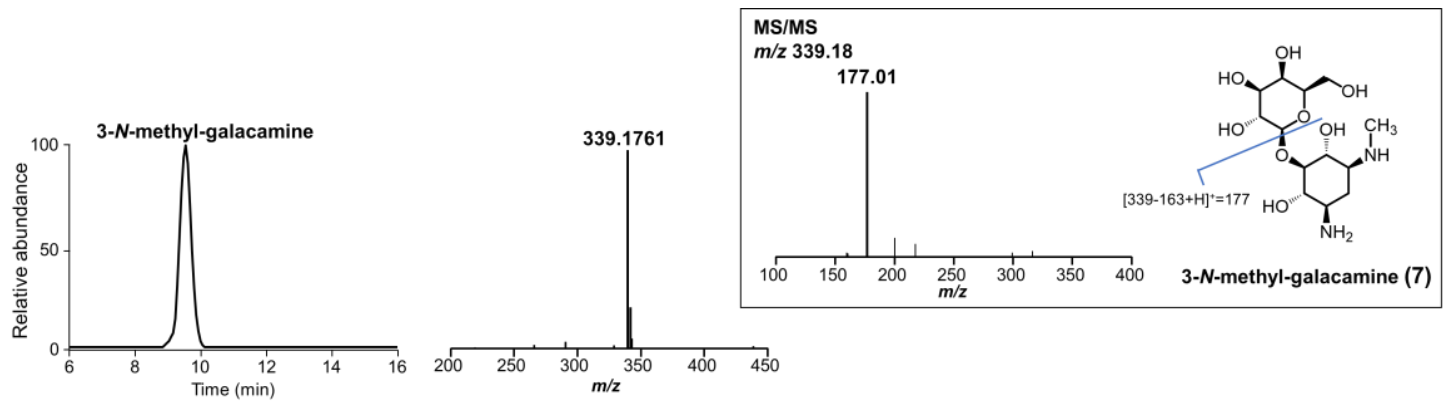
d. LC-ESI-HRMS and MS/MS Analysis of Talamine (4)

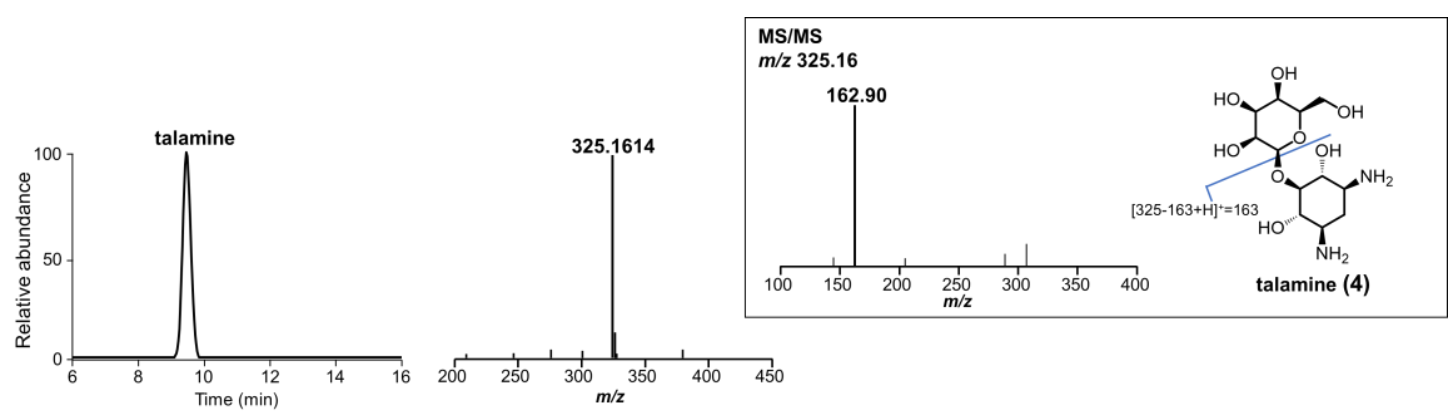

e. LC-ESI-HRMS and MS/MS Analysis of 3- N-demethyl-hygromycin B (2)

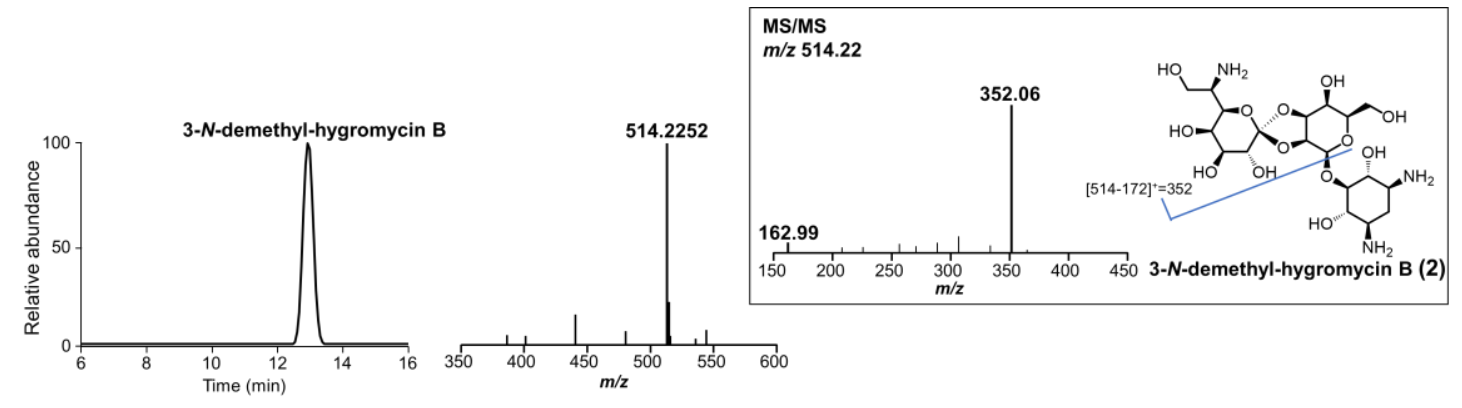

f. LC-ESI-HRMS and MS/MS Analysis of Hygromycin C (9)

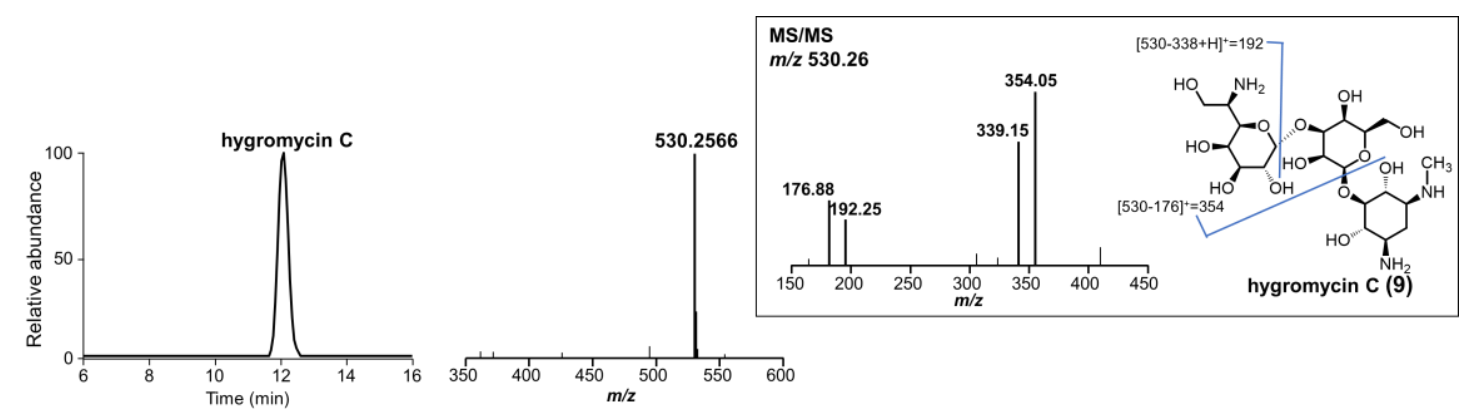

g. LC-ESI-HRMS and MS/MS Analysis of 3-N-demethyl-hygromycin C (10)

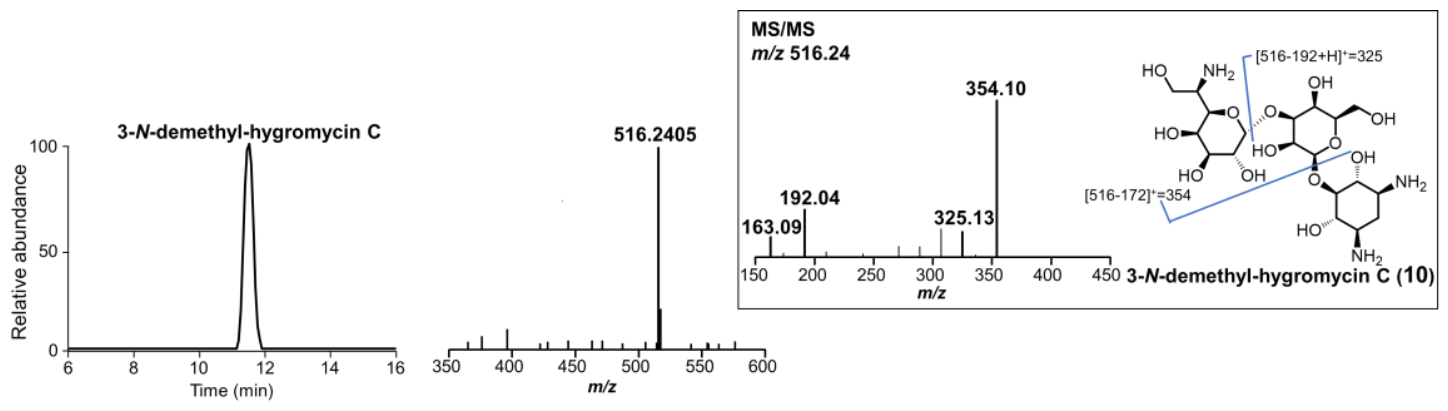


h. LC-ESI-HRMS and MS/MS Analysis of C-6"-deaminated-C-6"-hydroxyl-hygromycin C

(5)
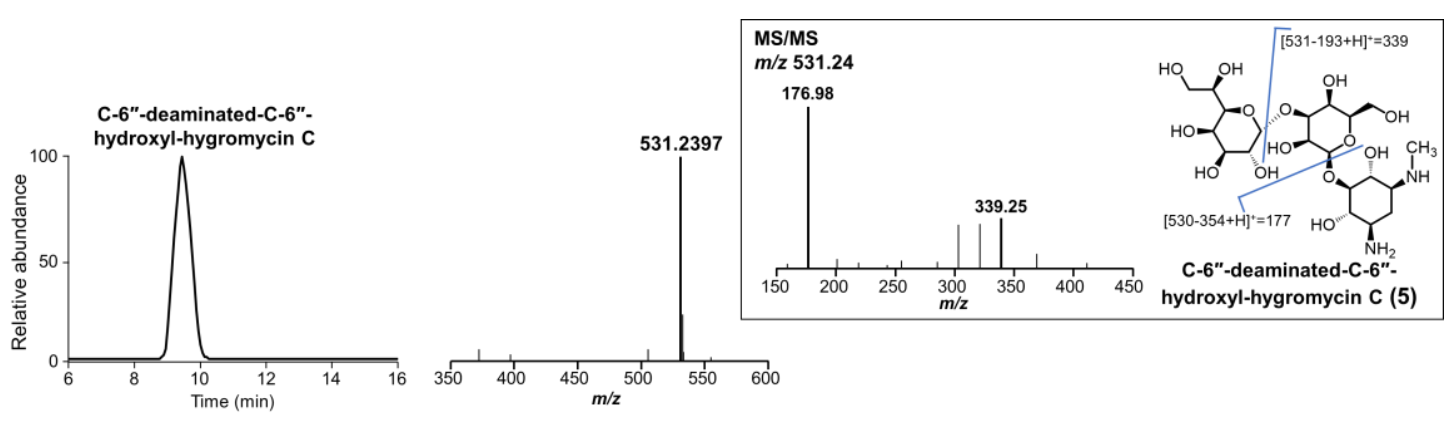

i. LC-ESI-HRMS and MS/MS Analysis of C-6"-deaminated-C-6"-hydroxyl-hygromycin B

(6)
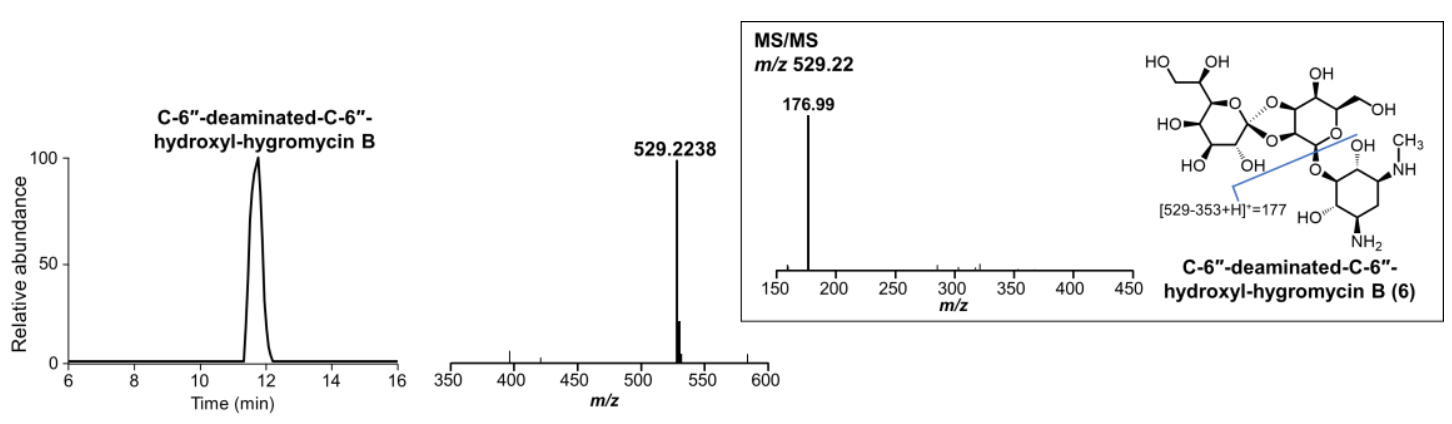

j. LC-ESI-HRMS and MS/MS Analysis of Galactamine (8)

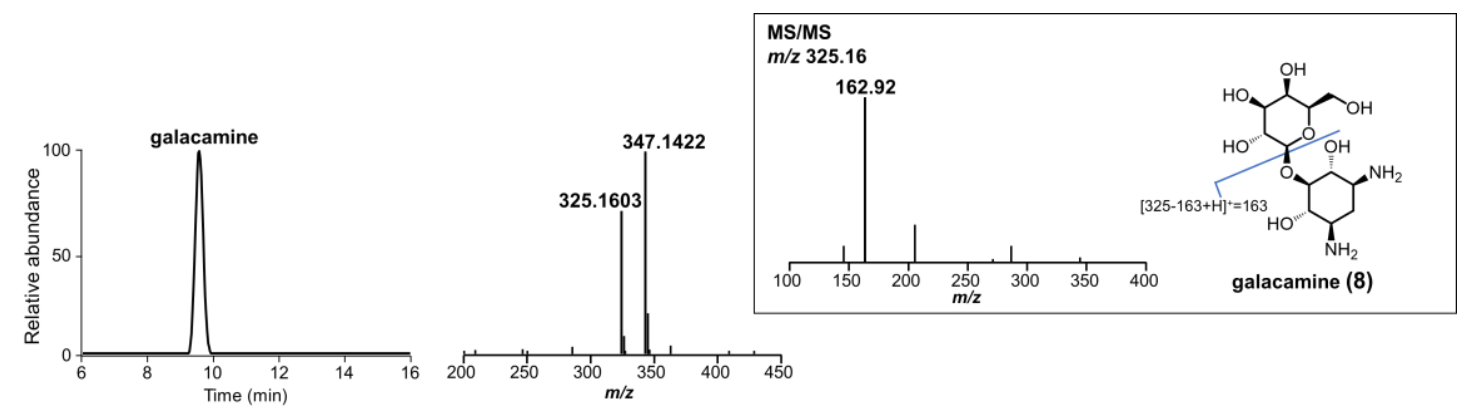


k. LC-ESI-HRMS and MS/MS Analysis of 2'-epi-hygromycin C (11)

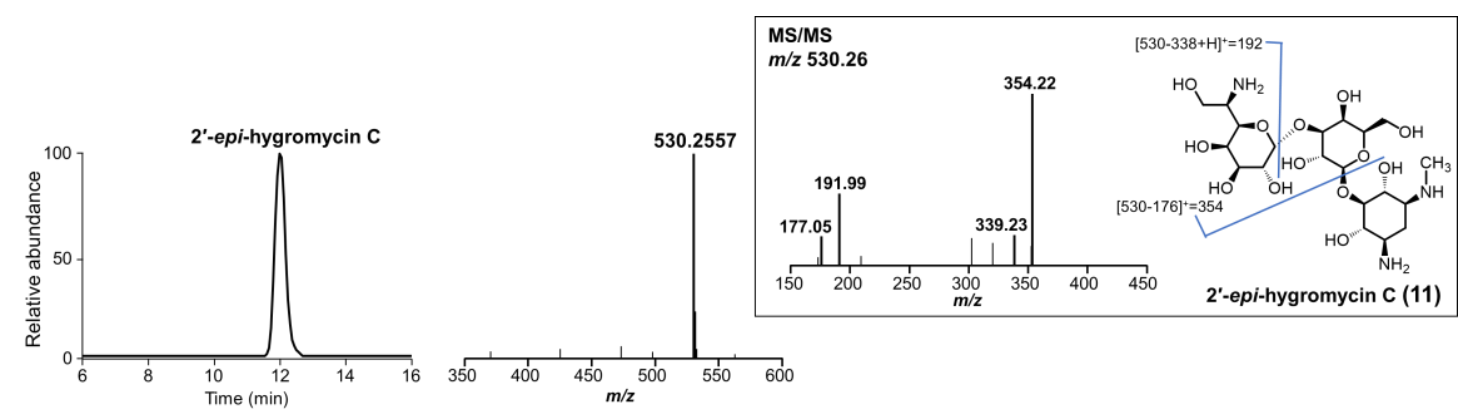

Figure S2. SDS-PAGE Analysis of Purified Recombinant Proteins Used in This

Study

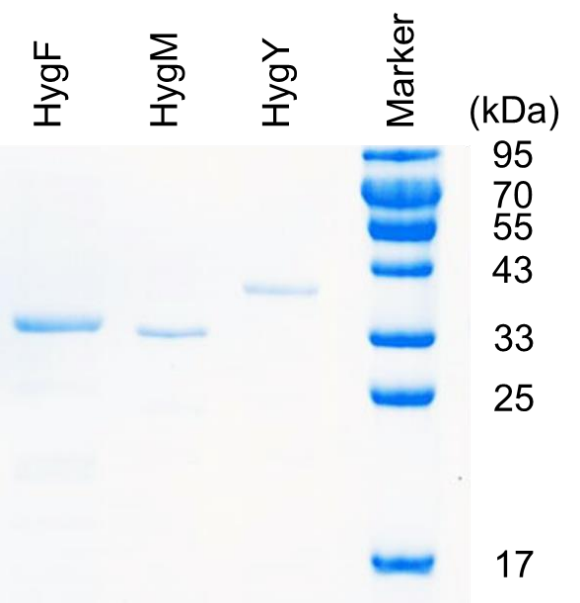

Gel electrophoresis was carried out under a voltage of $200 \mathrm{~V}$ for 60 min with $12 \%$ separation gel. 


\section{References}

(1) Huang, C., Huang, F., Moison, E., Guo, J., Jian, X., Duan, X., Deng, Z., and Sun, Y. (2015) Delineating the biosynthesis of gentamicin X2, the common precursor of the gentamicin C antibiotic complex. Chem. Biol. 22, 251-261.

(2) Zhong, Z., Guo, J., Deng, L., Chen, L., Wang, J., Li, S., Xu, W., Deng, Z. and Sun, Y. (2019). Base editing in Streptomyces with Cas9-deaminase fusions. bioRxiv, 630137.

(3) Li, S., Zhang, J., Liu, Y., Sun, G., Deng, Z., and Sun, Y. (2018) Direct genetic and enzymatic evidence for oxidative cyclization in hygromycin B biosynthesis. ACS Chem. Biol. 13, 2203-2210.

(4) MacNeil, D. J., Occi, J. L., Gewain, K. M., MacNeil, T., Gibbons, P. H., Ruby C. L., and Danis, S. J. (1992) Complex organization of the Streptomyces avermitilis genes encoding the avermectin polyketide synthase. Gene 115, 119-125. 